\title{
Arguments for transformational reform of mental health care for young people
}

\author{
P. McGorry* \\ Orygen, The National Centre for Excellence in Youth Mental Health, Parkville, Australia
}

Received 20 October 2014; Revised 16 November 2014; Accepted 19 November 2014; First published online 19 December 2014

Key words: Health service reform, youth mental health.

We have good reason to be concerned about the mental health of our young people. Emerging epidemiological evidence indicates that mental ill-health is now the key health issue facing young people world-wide (Bloom et al. 2011), contributing $45 \%$ of the burden of disability in those aged between 10 and 25 years (Gore et al. 2011). Furthermore, because the onset of mental illness peaks in late adolescence/early adulthood and subsequently impacts the most productive years of life (Kessler et al. 2005), it has recently been calculated to pose the greatest threat to the GDP of both the developed and developing nations over the next 20 years (Bloom et al. 2011).

Fortunately, the enormity of this problem, and the growing recognition of the scale of our neglect of it, has sparked a call to action among researchers, clinicians, service developers and policymakers world-wide, which has led to a growing international momentum to address the mental health needs of young people and their families. The need for transformational reform of mental health care, based on the principles of early intervention and with a priority focus on the developmental period of greatest need and capacity to benefit from investment - emerging adulthood - is increasingly being accepted world-wide, and has already led to the development of new models of care for our young people.

\section{Why is this new approach to youth mental health care so important?}

First, young people have the poorest access to mental health care of all age groups across the lifespan, largely as a result of poor awareness and help-seeking, structural and cultural flaws with the existing care systems, and a serious failure to recognise the importance of investment in youth mental health (McGorry, 2007; McGorry et al. 2013). Our primary health care system is

\footnotetext{
* Address for correspondence: Professor P. McGorry, AO, MD, Ph.D., FRACP, FRANZCP, Orygen, The National Centre for Excellence in Youth Mental Health, 35 Poplar Road, Parkville 3052, Australia.

(Email: pmcgorry@unimelb.edu.au)
}

largely designed to cater for physical health care needs, and is often seen as alienating by young people, with issues of accessibility, confidentiality and cost, as well as the organisation, location, milieu and even the decor of the services available being critical barriers to engagement (Ambresin et al. 2013). Our specialist system, divided as it is between the traditional child and adolescent and adult mental health services is another major barrier to care, not only owing to the differences in focus and therapeutic approach between the streams, but also because the discontinuity between these service streams falls right within the age range where the incidence of new onsets peaks (McGorry et al. 2011), and thus the system is weakest where it should be strongest (McGorry, 2007).

Second, young people in the early stages of a mental illness are a heterogeneous population, with varying and uncertain illness trajectories. They tend to present with blends of co-morbidities of variable intensity, particularly depression, anxiety, substance abuse and challenging personality traits that do not fit the existing diagnostic categories, and thus are all too often excluded from care by our current system. Moreover, the complexity and relative non-specificity of these symptom profiles means that different treatment approaches are required than those for full-threshold illness, with the emphasis being on offering care that is appropriate to the very early stages of illness, preemptive in nature, and with a strong preventive focus (McGorry et al. 2007).

Third, developmentally and culturally appropriate approaches are essential for the management of emerging disorders; young people's individual and group identity and their help-seeking needs and behaviours need to be central to any service model (McGorry, 2007). The available evidence shows that youth-specific services should be provided in an accessible, community-based, non-judgmental and non-stigmatising setting, where young people feel comfortable, have a say in how their care is provided, and can feel a sense of trust (Ambresin et al. 2013; McGorry et al. 2013). Ideally, this means creating a novel youth mental health model overlapping 
and linking with, but distinct in culture and expertise from, systems for younger children and older adults. Such a model will largely overcome both the issue of poor access to care and that of poor transition between the current service streams (Singh et al. 2010).

\section{What should a youth mental health service look like?}

Here, the challenge we face is one of scale, scope, culture, as well as expertise. Such a service stream needs to cover the entire spectrum of illness complexity and severity: from e-health, primary care services or enhanced primary care services for those with mild to moderate mental health issues, to specialised services for those with complex presentations or more severe illness. It also needs to acknowledge the unique developmental and socio-cultural needs of young people and offer care that is accessible, affordable, acceptable and appropriate to the stage of illness so that young people (and their families) can engage with it. Finally, it needs to offer care by clinicians with both the interest and expertise required to work with this sometimes difficult client group.

\section{Two examples}

Australia's headspace and Ireland's Headstrong are two different examples of such a service stream (McGorry et al. 2013). Although these two services were established by different mechanisms, have very different funding structures and operate under different models, they share the key features of a youth-friendly mental health service. Both are multidisciplinary enhanced primary care services, with close links to relevant local services frequently accessed by young people, and both provide easily accessible, free or lowcost care in a low-stigma, youth-friendly environment, with strong input at all levels from local young people. Both now offer significant national coverage, with headspace now running over 70 sites nation-wide in Australia, and with Headstrong's Jigsaw project opening 10 sites across Ireland. Both have lacked the back up and linkage with corresponding specialist youth mental health services and youth-friendly acute inpatient care since these are yet to be designed and funded. However, headspace is in the process of rolling out a national early psychosis programme to provide integrated specialist services for young people at ultra-high risk of, or experiencing, a first episode of psychosis, which it is hoped will ultimately be expanded to cater for the full diagnostic spectrum of potentially serious mental illness. Both Australia and Ireland also offer internet-based mental health support for young people, through eheadspace in Australia and ReachOut in Ireland. Together, these face-to-face and e-services have significantly increased young people's access to mental health care, whenever, wherever and however they choose to do so. Early indications suggest that better outcomes are occurring too (Cross et al. 2014). It is critical that as Ireland's economy recovers and new funding becomes available for health care and mental health reform and investment that growth funding is prioritised for this urgent and cost-effective focus to link with Headstrong. One hopes that Headstrong in turn will see the need for, be receptive to, and advocate for such reform, which must include cultural change.

\section{The future}

The long-term aim of these reforms is to develop a youth mental health stream that will provide a seamless coverage of mental health care from puberty to mature adulthood at around 25 years of age, with soft transitions on either side with child and adult mental health care. This system carefully adapts to the dynamics of biopsychosocial development and recognises the complexity and challenges faced by young people as they become independent adults, as well the burden of disease imposed on this age group by mental ill-health. It responds by blurring the distinctions between the tiers of primary and specialist care, including some aspects of acute care, in recognition of the complexity and sensitivity of the presentation of much of the mental ill-health apparent in young people. This allows for a flexible and appropriate response for each individual, depending on their own unique needs. Clearly, the success of these reforms will ultimately only be able to be assessed after careful evaluation, and evidently more health services and economic research is necessary to develop, refine, adapt and evaluate new service models, both within their individual contexts and cross-sectorally. However, the indications to date are that the model is justified, initially at least on the grounds of the significant improvement it offers in terms of access to care (Cross et al. 2014; Rickwood et al. 2014).

John Gunn, the eminent English forensic psychiatrist, once described the neglect of the mental health of young people as a form of 'self-harm' perpetrated by society upon itself (Gunn, 2004). Yet, if the silence and ignorance around this issue can be dispelled and solutions placed before the voting public, and especially parents and young people themselves, then in the foreseeable future this societal self-harm can be replaced by a potentially dramatic improvement in the mental health, well-being, productivity and fulfilment of young people. Transformational reform of mental health care should be based on the principles of early intervention and a priority focus on the developmental period of greatest need and capacity to benefit from investment: the period of emerging adulthood. The arguments for this type of transformational reform are 
resonating strongly with the community and with policymakers, and the number of young people endorsing them by voting with their feet and using these services speak for themselves (Cross et al. 2014; Rickwood et al. 2014). These examples of 21st century stigma free clinical culture will also facilitate some of the population-based and universal programs that may link with mental health awareness and promotion activities and with new internet-based technologies. If these new mindsets and reforms spread widely we may be able to reduce the lifelong impact of mental distress on our health, happiness and prosperity over the coming decades.

\section{Acknowledgement}

Professor McGorry would like to acknowledge the invaluable contributions of colleagues from the youth mental health movement world-wide, whose dedication and persistence has enabled these reforms to be put in place and the process of transformational change to begin.

\section{References}

Ambresin AE, Bennett K, Patton GC, Sanci LA, Sawyer SM (2013). Assessment of youth-friendly health care: a systematic review of indicators drawn from young people's perspectives. Journal of Adolescent Health 52, 670-681.

Bloom DE, Cafiero ET, Jane-Llopis E, Abrahams-Gessel S, Bloom LR, Fathima S, Fiegl AB, Mowafi M, Pandya A, Prettner K, Rosenberg L, Seligman B, Stein A, Weinstein C (2011). The Global Economic Burden of Non-Communicable Disease. World Economic Forum: Geneva.

Cross SP, Hermens DF, Hickie IB (2014). Treatment patterns and short-term outcomes in an early intervention youth mental health service. Early Intervention in Psychiatry, doi: 10.1111/eip.12191.
Gore FM, Bloem PJ, Patton GC, Ferguson J, Joseph V, Coffey C, Sawyer SM, Mathers CD (2011). Global burden of disease in young people aged 10-24 years: a systematic analysis. Lancet 377, 2093-2102.

Gunn J (2004). Foreword. In Adolescent Forensic Psychiatry (ed. S. Bailey and M. Dolan), p. xi. Arnold: London.

Kessler RC, Berglund P, Demler O, Jin R, Merikangas KR, Walters EE (2005). Lifetime prevalence and age-of-onset distributions of DSM-IV disorders in the National Comorbidity Survey Replication. Archives of General Psychiatry 62, 593-602.

McGorry PD (2007). The specialist youth mental health model: strengthening the weakest link in the public mental health system. Medical Journal of Australia 187, S53-S56.

McGorry P, Bates T, Birchwood M (2013). Designing youth mental health services for the 21st century: examples from Australia, Ireland and the UK. British Journal of Psychiatry. Supplement 54, s30-s35.

McGorry PD, Purcell R, Goldstone S, Amminger GP (2011). Age of onset and timing of treatment for mental and substance use disorders: implications for preventive intervention strategies and models of care. Current Opinion in Psychiatry 24, 301-306.

McGorry PD, Purcell R, Hickie IB, Jorm AF (2007). Investing in youth mental health is a best buy. Medical Journal of Australia 187, S5-S7.

Rickwood DJ, Telford NR, Parker AG, Tanti CJ, McGorry PD (2014). Headspace - Australia's innovation in youth mental health: who are the clients and why are they presenting? Medical Journal of Australia 200, 108-111.

Singh SP, Paul M, Ford T, Kramer T, Weaver T, McLaren S, Hovish K, Islam Z, Belling R, White S (2010). Process, outcome and experience of transition from child to adult mental healthcare: multiperspective study. British Journal of Psychiatry 197, 305-312. 